\title{
Pneumocystis jirovecii pneumonia in breast cancer mimicking SARS-COV-2 pneumonia during pandemic
}

\author{
Filippo Castelnuovo, Giorgio Tiecco, Samuele Storti, Benedetta Fumarola, \\ Nigritella Brianese, Davide Bertelli, Francesco Castelli \\ Division of Infectious and Tropical Diseases, University of Brescia and ASST Spedali Civili, Brescia, Italy \\ Article received 7 October, 2021; accepted 15 November, 2021
}

\section{SUMMARY}

Pneumocystis jirovecii pneumonia (PJP) is one of the most common HIV-related opportunistic infection. Apart from HIV patients, subjects treated with an associated therapy of high doses glucocorticoids and immunosuppressive drugs should be considered at risk. SARS-CoV-2 has become worldly known as the responsible of the pandemic that hit the world in late 2019 and that is still ongoing. Italy, and especially Brescia, was one of the area most struck by the pandemic, with a significant number of cases being reported (more than 112,648 as of October 2021).

The diagnosis of SARS-CoV-2 is mainly based on RTPCR assays performed on nasopharyngeal swab, X-ray of the chest and clinical manifestations.

We describe two cases of PJP in two immunocompromised patients with breast cancer who were admitted at Spedali Civili of Brescia hospital, Italy, with an in- itial diagnosis of SARS-CoV-2 pneumonia, despite testing negative to RT-PCR on nasopharyngeal swabs. We also retrospectively reassessed all cases of pneumonia deemed as SARS-CoV-2-related upon admission and then converted to PJP as the final diagnosis. We describe the two following cases to emphasize that clinicians should always be alert about PJP, even during the SARS-CoV-2 pandemic, and avoid focusing on COVID-19 exclusively. PJP should always be considered as a differential diagnosis in patients, particularly if immunosuppressed, with an X-ray or TC of the chest suggestive of interstitial pneumonia and a negative SARS-CoV-2 RT-PCR on nasopharyngeal swabs.

Keywords: Pneumocystis, breast cancer, SARS-CoV-2 pandemic.

\section{INTRODUCTION}

Dneumocystis jirovecii pneumonia (PJP) is main1 ly described in HIV-infected individuals as an AIDS-defining illness. At the beginning of the HIV epidemic, the documented rate of infection was as high as $20 \%$ among patients with less than 200 CD4+ T cells/ $\mu 1$ [1].

During the second COVID-19 pandemic wave, more than 6,000 patients with interstitial pneumonia were hospitalized at Spedali Civili of Brescia,

Corresponding author

Filippo Castelnuovo

E-mail: filippo.castelnuovo@tiscali.it
Italy. The accuracy and predictive values of SARSCoV-2 Nucleic Acid Amplification Tests (NAATs), including real-time polymerase chain reaction (RTPCR), have not been systemically evaluated. Reported false-negative rates have ranged from less than 5 to 40 percent, although these estimates are limited $[2,3]$. A recent meta-analysis that reviewed 50 studies comparing SARS-CoV-2 loads in nasopharyngeal (NP) swab and saliva samples, showed that the overall sensitivity of RT-PCR test from NP swab was $92 \%$ [4]. Considering these limitations in COVID-19's diagnosis, many interstitial pneumonia's cases admitted to the Spedali Civili of Brescia Hospital (Italy) were considered as SARSCoV-2 related even without a positive RT-PCR on nasopharyngeal swab, but only due to symptoms 
and radiological signs compatible with COVID-19 and/or to a previous contact with positive patients. Here we present a brief description of two cases of PJP in patients who were admitted to the Infectious and Tropical Disease Department, Spedali Civili of Brescia, Italy, with an initial diagnosis of COVID-19-related pneumonia, despite testing negative at SARS-CoV-2 RT-PCR on nasopharyngeal swab.

\section{CASES PRESENTATIONS}

Case A

In December 2020, a 65-years-old HIV-negative (CD4+ T cells 548 cells/ $\mu$ l) female presented to the Infectious and Tropical Diseases Department, Spedali Civili of Brescia, with fever and dyspnoea on for a day and was admitted with a suspected diagnosis of SARS-CoV-2 pneumonia. The patient was in treatment with neoadjuvant chemotherapy with paclitaxel and trastuzumab for a metastatic breast cancer (Her2+). Radiological pattern was extremely suggestive for SARS-CoV-2 pneumonia, as the chest X-ray and CT showed a subpleural and bilateral ground-glass pattern involving most of the lungs. Blood investigations were notable for raised inflammatory markers and decreased lymphocytes count. Despite these suggestive findings for COVID-19, she tested negative for SARS-CoV-2 RT-PCR and was managed with broad-spectrum intravenous antibiotics (piperacillin/tazobactam plus azithromycin) as per highrisk community acquired pneumonia. Despite the prompt treatment, her clinical conditions continued to deteriorate and continuous positive airway pressure (CPAP) (PEEP $10 \mathrm{cmH}_{2} \mathrm{O}, \mathrm{FiO}_{2} 50 \%$ ) was needed and carried on for 13 days.

Bronchoscopy with RT-PCR on bronchoalveolar lavage (BAL) was performed the day after her hospital admission and was hence necessary to make the correct diagnosis. The RT-PCR for SARS-CoV-2 was confirmed negative and Pneumocystis jirovecii was isolated. Specific treatment for PJP with high-dose trimethoprim-sulfamethoxazole (TMP-SMX) was started and patient's clinical conditions began to improve. This treatment was continued for 14 days, when the patient developed an allergic rash to the ongoing treatment. TMP-SMX was then substitute with weekly aerosol pentamidine. The patient was discharged five days after the last dose of TMP-SMX.

\section{Case B}

In December 2020, a 61-years-old HIV-negative (CD4+ T cells 657 cells/ $\mu 1)$ female presented to the Infectious and Tropical Diseases Department, Spedali Civili of Brescia, with a seven-days history of high fever and dry cough. Her medical history was significant for breast cancer, for which she was being treated with adjuvant chemotherapy with epirubicin. An arterial blood gas test on air confirmed respiratory failure with partial pressure of oxygen $\left(\mathrm{pO}_{2}\right)$ of $50 \mathrm{mmHg}$. As the clinical presentation, imaging was also suggestive for SARS-CoV-2 pneumonia: the X-ray and CT of the chest showed a ground-glass pattern involving the apex and the superior lobe of both lungs. Her conditions deteriorated quickly and High Flow Nasal Cannula (HFNC) was required. As in the former case, bronchoscopy with RT-PCR on bronchoalveolar lavage was performed as the RT-PCR on NP swab was confirmed negative for SARSCoV-2. However, RT-PCR on BAL was found positive for $P$. jirovecii and, once TMP-SMX was instituted, the patient's conditions got quickly better. She was discharged seven days after this correct therapy was started.

\section{DISCUSSION}

The two cases described above were admitted with an initial diagnosis of SARS-CoV-2 pneumonia, despite testing negative to RT-PCR on NP swabs. Trying to confirm that SARS-CoV-2 was the causative agent of their illness, they were both deeply investigated with BAL, but RT-PCR was confirmed negative for SARS-CoV-2 and resulted positive for $P$. jirovecii instead. Consequently to those two cases, we retrospectively reassessed all patients admitted to Spedali Civili of Brescia hospital for suspected SARS-CoV-2-related pneumonia at the time of admission and then eventually converted to PJP as final diagnosis. All data were retrieved from the hospital database where all the diagnosis of the discharged patients are collected.

$P$. jirovecii has been reclassified as a member of the fungi family upon cell wall composition and gene sequencing $[5,6]$. Patients with a significantly higher risk of developing PJP are those affected by haematologic malignancies, those who underwent hematopoietic cells or solid organ transplantation and subjects under treatment for cer- 
tain inflammatory or rheumatologic conditions. It is fair to say that patients with any condition that impairs cell-mediated immunity are likely more exposed to the risk of developing PJP. However, the major risk factor in HIV-negative subjects is the combined use of glucocorticoids and immunosuppressive therapy $[7,8]$.

Waks and Colleagues described nineteen cases of PJP in non-metastatic breast cancer patients over a period of thirteen years. All subjects who were diagnosed with PJP had been treated for a median of 64 days with either three or four cycles of adriamycin/cyclophosphamide (AC) chemotherapy and $16.4 \mathrm{mg}$ prednisone equivalents/day (median) as nausea prophylaxis. The overall incidence of PJP among 2057 patients subjected to the above therapeutic scheme was $0.6 \%$ (95\% confidence interval $0.3-1.0 \%$ ). The Authors identified a potential steroid dose/time threshold beyond which the risk of developing PJP increased and concluded that replacing dexamethasone and reducing the glucocorticoid dose of the supporting medication for chemotherapy-induced nausea could improve patients' safety [9].

In our clinic, from March 2020 to March 2021, a total of fifteen patients were diagnosed with PJP after being admitted with an initial diagnosis of suspected SARS-CoV-2 infection based on clinical symptoms and radiological signs. Indeed, none of them had a positive SARS-CoV-2 RT-PCR on NP swab when they were first admitted.

Nine patients had an underlying AIDS condition: two had a known chronic HIV-infection and seven were newly diagnosed. Median CD4 count, both absolute (40 cells $/ \mu \mathrm{L}$ vs 602 cells $/ \mu \mathrm{L}$ ) and percentage ( $5 \%$ vs $52.5 \%$ ), was significantly lower in those with AIDS than in the two cases presented above.

The remaining six patients included: two cases with breast cancer (presented above), one with a history of multiple myeloma treated with bone marrow transplantation, one who underwent renal transplantation and who was being treated with steroid therapy and selective inhibitor of m-TOR (everolimus), and two subjects on highdose steroid therapy due to rheumatic polymyalgia and a chronic obstructive pulmonary disease, respectively.

Median age was, respectively, 42-years-old in the HIV-positive patients' group and 63-years-old in the other group. Symptoms associated with
PJP comprised fever, non-productive cough, and dyspnoea on physical exertion and they were similar in HIV-positive and HIV-negative patients. Likewise, chest X-ray and CT images did not allow a clear differentiation between SARSCoV-2-pneumonia and PJP.

Considering that a misdiagnosis of PJP could occur even because HIV-infection and other causes of immunosuppression may be denied or unknown, clinicians must maintain a high level of clinical suspicion about PJP to establish a correct diagnosis.

Definitive diagnosis of $P$. jirovecii pneumonia requires detection and identification of the organism by RT-PCR assay on respiratory specimens collected with BAL, although a positive test is not able to differentiate colonization from infection.

In our case history, only three patients did not undergo bronchoscopy and PJP was diagnosed in accordance with ex-juvantibus criteria: one had a concomitant rheumatic polymyalgia and two were AIDS patients.

Although definitive diagnosis of PJP may not always be established, if a concrete clinical suspicion arises while assessing a high-risk patient, the specific treatment should be started, regardless of whether a definitive diagnosis has been made. A delayed start of the appropriate therapy might allow the disease to worsen to the point of respiratory failure.

One of the potential complications of PJP is acute respiratory distress syndrome (ARDS), which may require Intensive Care Unit access and invasive ventilation. In our case history, several patients with PJP needed auxiliary ventilation: three required mechanical invasive ventilation, and three were treated with non-invasive ventilation (NIV) in CPAP.

Four patients experienced TMP-SMX allergy and other agents were used for treatment, such as nebulized pentamidine or clindamycin plus primaquine.

All fifteen patients with PJP were discharged after a median of 27 days and no deaths occurred.

In conclusion it is the Authors' opinion that, even during the COVID-19 pandemic, clinicians should always maintain a high level of suspicion regarding all possible causes of interstitial pneumonia, including PJP, and avoid focusing exclusively on SARS-CoV-2-infection. Secondly, whenever an immunosuppressed patient presents with a nega- 
tive RT-PCR for SARS-CoV-2 on NP swab and an $X$-ray of the chest suggestive for interstitial pneumonia, invasive diagnostic manoeuvres, such as bronchoscopy, should be considered to establish the correct diagnosis.

\section{Conflicts of interest}

The Authors declare no conflict of interest.

\section{Ethic statements}

The patients' written consent was obtained and any information was anonymized as far as possible.

\section{Funding}

No funding was received for the development of this article.

\section{REFERENCES}

[1] Phair J, Munoz A, Detels R, Kaslow R, Rinaldo C, Saah A. The risk of Pneumocystis carinii pneumonia among men infected with human immunodeficiency virus type 1. Multicenter AIDS Cohort Study Group. N Engl J Med. 1990; 322 (3), 161-65.

[2] Weissleder R, Lee H, Ko J, Pittet MJ. COVID-19 di- agnostics in context. Sci Transl Med. 2020; 12 (546). doi: 10.1126/scitranslmed.abc1931.

[3] Long DR, Gombar S, Hogan CA, et al. Occurrence and Timing of Subsequent SARS-CoV-2 RT-PCR Positivity Among Initially Negative Patients. medRxiv Prepr Serv Heal Sci. 2020; doi: 10.1101/2020.05.03.20089151.

[4] Ibrahimi N, Delaunay-Moisan A, Hill C, et al. Screening for SARS-CoV-2 by RT-PCR: Saliva or nasopharyngeal swab? Rapid review and meta-analysis. PLoS One. 2021; doi: 10.1371/journal.pone.0253007.

[5] Amber KT. Balancing the risks and benefits of prophylaxis: a reply to "Pneumocystis jiroveci pneumonia in patients treated with systemic immunosuppressive agents for dermatologic conditions". Int J Dermatol. 2017; 56 (1), 4-5.

[6] Fillâtre P, Revest M, Belaz S, et al. Pneumocystosis in non-HIV-infected immunocompromised patients. Rev Med Interne. 2016; 37 (5), 327-36.

[7] Masur H. HIV-Related Opportunistic infections are still relevant in 2015. Top Antivir Med. 2015; 23 (3): 116-19. [8] Rey A, Losada C, Santillán J, et al. Pneumocystis jiroveci infection in patients with and without HIV: A comparison. Rev Chilena Infectol. 2015; 32 (2), 175-80.

[9] Waks AG, Tolaney SM, Galar A, et al. Pneumocystis jirovecipneumonia (PCP) in patients receiving neoadjuvant and adjuvant anthracycline-based chemotherapy for breast cancer: incidence and risk factors. Breast Cancer Res Treat. 2015; 154, 359-67. 